\title{
A comparison of shear bond strength of orthodontic brackets bonded with four flowable composites bonded under contamination: An Ex-Vivo study
}

\author{
Sunil Kumar M., Mahantesh C., Umesh H. M., Ashita Talwar, Sufia Qaiser, Sonal Sahasrabudhe \\ Department of Orthodontics \& Dentofacial Orthopaedics, M. S. Ramaiah Dental College \& Hospital, M. S. Ramaiah University of Applied \\ Sciences, Faculty of Dental Sciences, Bangalore, India
}

Email address:

drlinus1978@gmail.com (Sunil K. M.),dr.mahantesh9@gmail.com (Mahantesh C.),drhmumesh@gmail.com (Umesh H. M.), ashiat2097@gmail.com (Ashita T.), sufiaqaiser25@gmail.com (Sufia Q.), sonalsahasrabudhe15@gmail.com (Sonal S.)

\section{To cite this article:}

Sunil Kumar M., Mahantesh C., Umesh H. M., Ashita Talwar, Sufia Qaiser, Sonal Sahasrabudhe. A Comparison of Shear Bond Strength of Orthodontic Brackets Bonded with Four Flowable Composites Bonded under Contamination: An Ex-Vivo Study. International Journal of Materials Science and Applications. Vol. 4, No. 1, 2015, pp. 47-51. doi: 10.11648/j.ijmsa.20150401.19

\begin{abstract}
Objective: To evaluate the shear bond strength of the brackets bonded with adhesive and flowable composites under contamination by human blood and human saliva\& to evaluate enamel damage post debonding using ARI index. Material and Methods: 100 human premolars extracted for orthodontic reasons were used \& shear bond strengths of 4 flowable composites and one conventional composite were evaluated under 2 bonding conditions: (1) contamination with saliva (2) contamination with blood. Contamination was done after the application of primer in all the groups and before bonding the brackets, decontamination was done by using the air water spray for 5 seconds and air drying the surface. Shear bond strengths were measured with universal testing machine after placing the bonded specimen in deionized water and incubator at $37^{\circ} \mathrm{C}$ for 72 hours to permit adequate water absorption and equilibration. After debonding, each specimen was examined under a stereoscopic zoom microscope (10X) to identify the location of the bond failure. The residual composite remaining on the premolar was assessed by using the remnant index (ARI), where each specimen was scored according to the amount of material remaining on the enamel surface. Results: conventional composite showed the highest bond strength in both blood and salivary contaminations. Among the flowable composites, each behaved differently. Blood contamination showed reduced bond strengths in all the groups. Decontamination with air water spray and air drying the surface before bonding the brackets seems to be satisfactory. Conclusion: with use of a primer, flowable composites can be used to bond the orthodontic brackets under contamination and decontamination with air water spray for 5 seconds and air drying the surface before bonding seems to be satisfactory if contamination occurs after the application of the primer.
\end{abstract}

Keywords: Shear Bond Strength, Flowable Composites, Contamination

\section{Introduction}

The acid etching technique was first introduced by Buonocore in 19551 and this technique was used by the Newman ${ }^{1}$ in 1965 to bond the orthodontic brackets. This process is colloquially known as 'bonding'. During bonding enamel surface contamination can occur at two critical stages of the bonding procedure: after the tooth surface has been etched and after the primer has been applied ${ }^{2}$. Following salivary contamination, a biofilm forms over the etched enamel ${ }^{3}$. Thus, most of the pores become blocked, and the penetration of resin is altered, resulting in resin tags insufficient in number and length ${ }^{4}$.
Among the composite resins that could be used in orthodontics as bonding agents today, flowable composite merits great attention because of its clinical handling characteristics.This includes fluid injectability and nonstickiness which enabled the material to be packed or condensed. The development of flowable composites was based upon its flowable viscosity and not any clinical evidence of success for specific applications ${ }^{5}$.

During bonding enamel surface contamination can occur at two critical stages of the bonding procedure: after the tooth surface has been etched and after the primer has been applied $^{2}$.

Contamination of the enamel surface therefore requires the 
etching procedure to be repeated to ensure the adequate bonding of composites. For this reason several studies have evaluated the effects of contaminants like water, saliva, and blood on shear bond strength ${ }^{6}$.

Keeping in mind all the above mentioned points, this study is aimed to evaluate the shear bond strength of the brackets bonded with adhesive and flowable composites under contamination by human blood and human saliva.

\section{Material and Methods}

100 human maxillary permanent premolars extracted for orthodontic purpose were collected from the Department of Oral and Maxillofacial surgery at M.S.Ramaiah Dental College and Hospital, Bangalore.

\subsection{Methodology}

A total of 100 sound-extracted human premolars were stored in $0.5 \%$ chloramine $\mathrm{T}$ solution after extraction. The buccal crown surface of each premolar were rinsed and dried after a 15 -second polish with fluoride free pumice slurry.

100 teeth were divided into 5 groups with 20 teeth in each group $(\mathrm{N}=20)$ and 4 groups were considered as experimental groups and were used to test shear bond strength of flowable composites $(\mathrm{N}=20 \times 4=80)$ and one group $(\mathrm{N}=20)$ were used to test bond strength of conventional composite, which were considered as the control group.

The buccal enamel surfaces were etched with $37 \%$ phosphoric acid gel (Scotch Bond) for 30 seconds, rinsed for 15 seconds, and dried with oil-free and moisture-free air for 20 seconds until the enamel became faintly white.

After acid etching, a thin layer of Transbond XTprimer was applied, and each experimental group were divided into 2 subgroups $(\mathrm{N}=10)$ and four flowable composites (Esthet $\mathrm{X}$ Flow, (Dentsply); Admira, (Voco); Helioset Orthodontic, (IvaclorVivadent); Restofil N Flow, (Anabond) and a conventional orthodontic bonding system Transbond XT( 3M ESPE), were examined.

After the application of primer, half of the teeth $(\mathrm{N}=10)$, in each experimental group were exposed to $0.01 \mathrm{ml}$ of human blood and then air water spray was used for 5 seconds to decontaminate the surface. Compressed oil free air was used to dry the surface. The blood was collected from the researcher.

One hundred stainless steel metal premolar brackets with a micro-etched base (3M Gemini, 0.022 slot M.B.T. premolar brackets) were directly bonded to the acid etched enamel. Composite materials was applied to the base of the brackets, the brackets were then oriented with their bases parallel to the floor and pressed firmly against the enamel surface, and excess removed with an explorer. The samples were light cured (3M ESPE, ELISPAR 2500) for 20 seconds, (10 seconds mesially and 10 seconds distally).

The same procedure was done with the other half of each experimental group $(\mathrm{N}=10)$, but using human saliva. The saliva was collected from a donor who was instructed to brush teeth and refrain from eating for 1hour so that saliva could be collected. Saliva was collected immediately before the contamination procedure. Fig. 1 shows sample of mounted teeth.

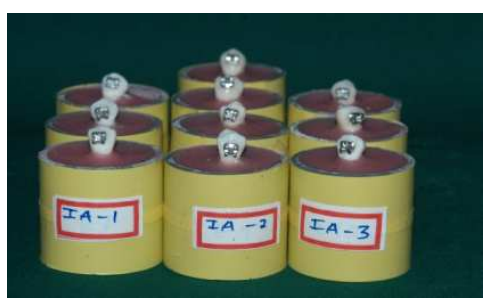

Fig. 1. Sample of mounted teeth

The bracket teeth were then immersed in sealed containers of deionized water and placed in an incubator at $37^{\circ} \mathrm{C}$ for 72 hours to permit adequate water absorption and equilibration, and then the tests for examining the shear bond strength were performed.

The specimens were secured in a jig attached to the base plate of a universal testing machine. A looped ligature wire was mounted in the movable crosshead of the testing machine and positioned so that the leading edge were aimed at the enamel-composite interface before being brought into contact. A crosshead speed of $0.5 \mathrm{~mm} / \mathrm{min}$ was used.(fig.2)

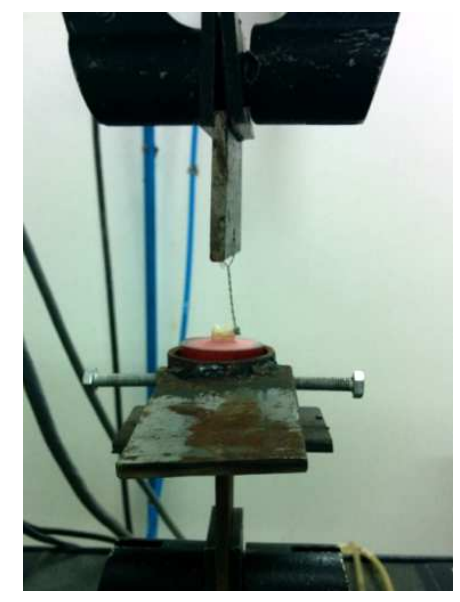

Fig. 2. Specimen attached to jig in universal testing machine

After debonding, each specimen was examined under a stereoscopic zoom microscope (10X) to identify the location of the bond failure. The residual composite remaining on the premolar was assessed by using the remnant index (ARI), where each specimen was scored according to the amount of material remaining on the enamel surface ${ }^{9}$.

\subsection{Statistical Analysis}

Descriptive and inferential statistical analysis has been carried out in the present study. Results on continuous measurements are presented on Mean \pm SD (Min-Max) and results on categorical measurements are presented in Number (\%). Significance is assessed at $5 \%$ level of significance.

Analysis of variance (ANOVA) has been used to find the significance of study parameters between three or more groups, Student $t$ test ( two tailed, independent)has been used 
to find the significance of study parameters on continuous scale between two groups (Inter group analysis) on metric parameters. Chi-square/ Fisher Exact test has been used to find the significance of study parameters on categorical scale between two or more groups

\section{Results}

\subsection{Shear Bond Strength}

All the groups in this study showed clinically acceptable bond strengths, both under salivary and blood contaminations. The differences in shear bond strengths of all the flowable composites under blood and salivary contamination were statistically significant; whereas that of conventional composite was not statistically significant.
Under salivary contamination, Group IA (Transbond XT) produced the highest value of $17.69 \pm 2.21 \mathrm{MPa}$ followed by group VA (Esthet X Flow) with $17.26 \pm 2.28 \mathrm{Mpa}$, followed by group IIA (Admira Flow) 16.36 \pm 1.58 , followed by IVA (Restofil N Flow Nano) 16.20 \pm 2.32 Mpa \& group IIIA (Heliosit Orthodontic) $12.85 \pm 1.79 \mathrm{MPa}$ respectively.

Under blood contamination Group IB (Transbond XT) produced the highest value of $15.55 \pm 2.81 \mathrm{MPa}$ followed by group IIB (Admira Flow) with $13.24 \pm 2.02 \mathrm{Mpa}$, followed by group VB (Esthet X Flow) $12.36 \pm 3.26 \mathrm{MPa}$, followed by IIIB (Heliosit Orthodontic $\neg$ ) $10.92 \pm 2.12 \mathrm{MPa} \&$ group VB (Restofil N Flow Nano) 10.50 $\pm 2.20 \mathrm{MPa}$ respectively.

ANOVA test revealed statistically significant difference between 5 groups with Salivary Contamination and blood contamination as shown in table 1 .

Table 1. Comparison of shear bond strength in salivary and blood contamination for Transbond XT, Admira Flow, Heliosit orthodontic, Restofil N Flow Nano and Esthet X Flow.

\begin{tabular}{|c|c|c|c|c|c|c|c|c|c|c|}
\hline \multirow{2}{*}{ ARI } & \multicolumn{2}{|c|}{ Transbond XT } & \multicolumn{2}{|c|}{ Admira Flow } & \multicolumn{2}{|c|}{ Heliosit orthodontic } & \multicolumn{2}{|c|}{ Restofil N Flow Nano } & \multicolumn{2}{|c|}{ Esthet X Flow } \\
\hline & Saliva & Blood & Saliva & Blood & Saliva & Blood & Saliva & Blood & Saliva & Blood \\
\hline $\begin{array}{l}\text { Mean } \\
\text { P value }\end{array}$ & $\begin{array}{l}17.69 \pm 2.21 \\
0.085\end{array}$ & $15.55 \pm 2.81$ & $\begin{array}{l}16.36 \pm 1.58 \\
0.001\end{array}$ & $13.24 \pm 2.02$ & $\begin{array}{l}12.85 \pm 1.79 \\
0.042\end{array}$ & $10.92 \pm 2.12$ & $\begin{array}{l}16.20 \pm 2.32 \\
0.000\end{array}$ & $10.57 \pm 2.20$ & $\begin{array}{l}17.26 \pm 2.28 \\
0.001\end{array}$ & $12.36 \pm 3.26$ \\
\hline
\end{tabular}

\subsection{Adhesive Remnant Index (ARI) Comparisons}

The frequency distribution of ARI scores of the 5 adhesive systems under salivary and blood contamination.

The results of Fischer test indicated a statistically insignificant difference $(\mathrm{P} \geq 0.05)$ in the ARI scores between the adhesives with salivary contamination and with blood contamination.

For all adhesive systems, no statistically significant difference $(\mathrm{P} \geq 0.05)$ was obtained within sample with salivary and blood contamination as shown in table (2).

Table 2. Comparison of ARI score in salivary and blood contamination for Transbond XT, Admira Flow, Heliosit orthodontic, Restofil N Flow Nano and Esthet X Flow.

\begin{tabular}{|c|c|c|c|c|c|c|c|c|c|c|}
\hline \multirow{2}{*}{ ARI } & \multicolumn{2}{|c|}{ Transbond XT } & \multicolumn{2}{|c|}{ Admira Flow } & \multicolumn{2}{|c|}{ Heliosit orthodontic } & \multicolumn{2}{|c|}{ Restofil N Flow Nano } & \multicolumn{2}{|c|}{ Esthet X Flow } \\
\hline & Saliva & Blood & Saliva & Blood & Saliva & Blood & Saliva & Blood & Saliva & Blood \\
\hline Nil & $4(40 \%)$ & $3(30 \%)$ & $6(60 \%)$ & $7(70 \%)$ & $9(90 \%)$ & $7(70 \%)$ & $6(60 \%)$ & $6(60 \%)$ & $7(70 \%)$ & $7(70 \%)$ \\
\hline 1.00 & $4(40 \%)$ & $6(60 \%)$ & $3(30 \%)$ & $3(30 \%)$ & $1(10 \%)$ & $3(3 \%)$ & $3(30 \%)$ & $4(40 \%)$ & $3(30 \%)$ & $3(30 \%)$ \\
\hline 2.00 & $2(20 \%)$ & $1(10 \%)$ & $1(10 \%)$ & $0(0 \%)$ & $0(0 \%)$ & $0(0 \%)$ & $1(10 \%)$ & $0(0 \%)$ & $0(0 \%)$ & $0(0 \%)$ \\
\hline 3.00 & $0(0 \%)$ & $0(0 \%)$ & $0(0 \%)$ & $0(0 \%)$ & $0(0 \%)$ & $0(0 \%)$ & $0(0 \%)$ & $0(0 \%)$ & $0(0 \%)$ & $0(0 \%)$ \\
\hline Total & $10(100 \%)$ & $\begin{array}{l}10 \\
(100 \%)\end{array}$ & $\begin{array}{l}10 \\
(100 \%)\end{array}$ & $\begin{array}{l}10 \\
(100 \%)\end{array}$ & $\begin{array}{l}10 \\
(100 \%)\end{array}$ & $\begin{array}{l}10 \\
(100 \%)\end{array}$ & $\begin{array}{l}10 \\
(100 \%)\end{array}$ & $\begin{array}{l}10 \\
(100 \%)\end{array}$ & $\begin{array}{l}10 \\
(100 \%)\end{array}$ & $\begin{array}{l}10 \\
(100 \%)\end{array}$ \\
\hline
\end{tabular}

\section{Discussion}

Among the composite resins that could be used in orthodontics as bonding agents today, flowable composite merits great attention because of its clinical handling characteristics. This includes fluid injectability and nonstickiness which enabled the material to be packed or condensed. The development of flowable composites was based upon its flowable viscosity and not any clinical evidence of success for specific applications.

These properties were a result of low viscosity of the resin obtained by reducing the filler concentration of traditional hybrid composites but maintaining the same filler size. Depending on the type of filler used, the majority of flowable composites are filled between $41-53 \%$ by volume which translates into $56-70 \%$ by weight. Most manufacturers will cite filler content by weight because the number is always higher. ${ }^{5}$
Conventional bonding system uses three different agents on the enamel conditioner, a primer solution and an adhesive resin for the process of bonding orthodontic brackets to enamel. The use of primer was an essential part of the bonding procedure of composite adhesives to allow good wetting and penetration of the sealant into the etched enamel surface ${ }^{8}$.

Many studies have been done on the flowable composites and various parameters like suitability of the materials for orthodontic bracket bonding 5,10 , polymerization shrinkage, elasticity, as filled adhesives, flow and flexural strength ${ }^{11}$, Contraction stress, elastic modulus, and degree of conversion $^{12}$. From the above mentioned studies it was concluded that the flowable composites can be used for bonding orthodontic brackets without the application of the primer. One study ${ }^{13}$, measured the shear bond strength of the flowable composites with the application of the primer prior 
to bonding the brackets with flowable composites, and found that the shear bond strength increased with the use of primer along with the flowable composites.

But no studies have been done using the flowable composites under contamination. Therefore this study was undertaken to evaluate the shear bond strength of 4 different flowable composites under salivary and blood contamination and decontamination, in comparison with conventional composite, and also assess the bracket failure mode using the ARI index. Since all the previous studies looked at different variables $5,10,11,12,13$, comparison with our results is impossible.

The most common contaminants of enamel during bonding procedures are saliva and blood. Whereas saliva occurs in all bonding situations, blood is mainly a problem when rebonding brackets if there is gingivitis ${ }^{14}$.

When contamination occurs with blood, it seems that high amounts of organic substances impede the binding between primer and adhesive ${ }^{15}$. Therefore protection of the bonding sites from blood contamination is therefore essential, especially for surgical exposure of retained teeth. Complete repetition of all bonding steps would again lead to optimal adhesion $^{16}$.

However, repeated etching would also cause further enamel loss. A decontamination procedure that does not involve repeated etching would therefore be preferable. We found that cleaning a contaminated surface with water and air and air drying the surface was sufficient to obtain adequate bonding forces. For all composite groups in this study, bond strength after decontamination without repeated etching was optimal. Our results conclude with the studies done by Ghavam $^{17}$ and Sayinsu et $\mathrm{al}^{18}$. A direct comparison is not possible because of different methodologies involved.

In this study, all the composites exhibited higher bond strength than the optimal bond strength needed for orthodontic purposes that was recommended by Reynolds ${ }^{19}$.

Though the bond strengths produced by all the composites in this study were higher than the recommended values, the bond strength produced under blood contamination were significantly lower than the ones produced under salivary contamination. This finding concludes with studies by Cacciafesta $^{20}$, Ozoptrak ${ }^{9}$, which say that contamination with blood led to uniformly and highly decreased bonding forces.

In our studies no significant difference in ARI scores was recorded for any adhesive under salivary and blood contaminations. This might indicate that ARI scores depend on complicated factors ${ }^{21}$.

Composite resin content fillers are added to improve strength, increase stiffness, reduce dimensional changes, and improve handling. However, the best mechanical properties could be achieved by incorporating high concentrations of filler particle of various sizes into the resin.

Faltermeier et $\mathrm{al}^{22}$ found that the filler level influences the bond strength of orthodontic brackets, because higher filled adhesives seem to provide greater bond strength than do lower filled or unfilled resins. This explains the reason why the bond strengths of the Heliosit Orthodontic group produced lower bond strengths when compared to other flowable composites and the reason for gaining the highest bond strengths with Transbond XT.

Heliosit Orthodontic is a flowable composite intended for bonding orthodontic brackets and therefore contains only $14 \%$ highly dispersed silicone as filler particles according to manufacturer's information. Whereas Transbond XT is conventional orthodontic composite and contains around $70-80 \%$ filler content according to manufacturer's information.

The results obtained with Esthet X Flow also can be justified based on the filler content. Esthet X Flow is a micro hybrid composite with filler content upto $61 \%$ by weight according to manufacturer's information.

In this context, clinicians should consider that polymerization shrinkage increases as the filler content decreases and this may cause formation of microleakagepromoting microgaps between the adhesive and the enamel surface, which might initiate the undesirable effect of white spot lesions. The mechanical properties in composite resins with high concentrations of filler particles of various sizes have shown better results ${ }^{23}$.

\section{Conclusions}

Our investigation showed that decontamination consisting of thorough rinsing with air and water, and drying with air can be used successfully on surfaces contaminated with blood or saliva after priming. Our study also concludes that application of primer significantly increases the bond strength of the flowable composites and the bond strength of the flowable composites increases with the filler content. Transbond XTTM adhesive, which is a conventional orthodontic composite, has superior shear bond strength than the flowable composites used in this study. Esthet X Flow, which is micro hybrid restorative flowable composite, exhibited highest bond strength among the flowable composites used in this study. Heliosit Orthodontic, a composite designed for bonding the orthodontic brackets produced the lowest bond strengths among the flowable composites used in this study. But the shear bond strength produced by this composite were above the minimum values recommended for orthodontic bracket bonding. Admira Flow which is a Ormocer, and Restofil N Flow Nano which is a nano hybrid restorative composite, can be used for bonding the orthodontic brackets. Contamination with blood during orthodontic procedures significantly decreases the shear bond strength of the adhesives. The bond failure for both conventional and flowable composites appeared to be same. Clinical management of contaminated bonding surfaces can be recommended as follows: renewed etching and priming is not necessary. Simple decontamination with water air spray and air drying the surface gives sufficient bond strength if contamination occurs after the application of the primer.

We thank the manufacturers, and people who helped us in conducting the shear bond strength and ARI index for this study. 


\section{References}

[1] Newman G.V: Epoxy adhesion for orthodontic attachment. Progress report. Am J of OrthodDentofacialOrthop 1965:51(12):901-12.

[2] Sfondrini MF, Cacciafesta V, Scribante A, De Angelis M, Klersy C. Effect of blood contamination on shear bond strength of brackets bonded with conventional and selfetching primers. Am J of OrthodDentofacialOrthop. 2004; 125:357360.

[3] Silverstone LM, Hicks MJ, Featherstone MJ. Oral fluid contamination of etched enamel surfaces: a SEM study. J Am Dent Assoc. 1985;110:329-332

[4] Hormati AA, Fuller JL, Denehy GE. Effects of contamination and mechanical disturbance on the quality of acid-etched enamel. J Am Dent Assoc. 1980; 100:34-38.

[5] Elaut J, Asscherickx K, VandeVannet B, Wehrbein $H$. Flowable composites for bonding lingual retainers. J ClinOrthod. 2002; 36:597-8.

[6] Webster MJ, Nanda RS, Duncanson MG, Khajotia SS, Sinha PK. The effect of saliva on shear bond strengths of hydrophilic bonding systems. Am J of OrthodDentofacialw233 Orthop 2011; 119:54-58.

[7] Artun J, Bergland S. Clinical trials with crystal growth conditioning as an alternative to acid etch enamel treatment. Am J Orthod 1984; 85: 333-40.

[8] Barkmeier WW, Erickson RL et al: Shear bond strength of composite to enamel and dentine using Scotchbond multipurpose. Am J Dent 1994: 175-9

[9] Oztoprak MO, Isik F, Sayinshu K, Arun T, Aydemir B. Effect of blood and saliva contamination on shear bond strength of brackets bonded with 4 adhesives. Am J of OrthodDentofacial Orthop 2007'131:238-242

[10] TancanUysal, Zafer Sari, and Abdullah Demir.Are the Flowable Composites Suitable for Orthodontic Bracket Bonding?. The Angle Orthodontist: October 2004, Vol. 74, No. 5, pp. 697-702

[11] Dong-Bum Ryou, Hyo-Sang Park, Kyo-Han Kim, and TaeYub Kwon. Use of Flowable Composites for Orthodontic Bracket Bonding. The Angle Orthodontist: November 2008, Vol. 78, No. 6, pp. 1105-1109.
[12] Cadenaro M, Codan B, Navarra CO, Marchesi G, Turco G, Di Lenarda R, Breschi L. Contraction stress, elastic modulus, and degree of conversion of three flowable composites. Eur J Oral Sci. 2011 Jun;119(3):241-5.

[13] Albaladejo A, Montero J, Gómez de Diego R, López-Valverde A. Effect of adhesive application prior to bracket bonding with flowable composites. Angle Orthod. 2011 Jul;81(4):71620

[14] Lorenz Brauchli, Martina Eichenberger, Markus Steineck, and Andrea Wichelhaus. Influence of decontamination procedures on shear forces after contamination with blood or saliva. Am J of OrthodDentofacialOrthop 2010 Oct; 138(4):435-41.

[15] Silverman E, Cohen M, Demke RS, Silverman M. A new light cured glass ionomer cement that bonds brackets to teeth without etching in the presence of saliva. Am J Orthod Dentofacial Orthop 1995; 108:231-6.

[16] Hormati AA, Fuller JL, Denehy GE. Effects of contamination and mechanical disturbance on the quality of acid-etched enamel. J Am Dent Assoc 1980; 100:34-8.

[17] Neelagiri K, Kundabala M, Shashi RA, Thomas MS, Parolia A.Effects of saliva contamination and decontamination procedures on shear bond strength of self-etch dentine bonding systems: An in vitro study. J Conserv Dentistry 2010; 13:71-5.

[18] Sayinsu K, Isik F, Sezen S, Aydemir B. Effect of blood and saliva contamination on bond strength of brackets bonded with a protective liquid polish and a light-cured adhesive. Am J Orthod Dentofacial Orthop 2007; 131:391-4.

[19] Reynolds IR. A review of direct orthodontic bonding. Br J orthod1975; 2:171-8

[20] Cacciafesta V, Sfondrini MF, Scribante A, De Angelis M, Klersy C. Effect of blood contamination on shear bond strength of brackets bonded with a self-etching primer combined with a resin-modified glass ionomer. Am J Orthod Dentofacial Orthop 2004; 126:703-8.

[21] Sunder Rao P S S , Richard J : An Introduction to Biostatistics, A manual for students in health sciences, New Delhi: Prentice hall of India. 86-160.

[22] Faltermeier A, Rosentritt M, Faltermeier R, Reicheneder C, Mussig D. Influence of filler level on the bond strength of orthodontic adhesives. Angle Orthod. 2007;77:494-8.

[23] Ostertag AJ, Dhuru VB, Ferguson DJ, Meyer RA. Shear, torsional, and tensile bond strengths of ceramic brackets using three adhesive filler concentrations. Am J Orthod Dentofacial Orthop.1991;100:251 